De acuerdo con los resultados obtenidos, a pesar de ser segura y bien tolerada, la inmunoterapia con una única dosis de SRL172 como adyuvante de la quimioterapia antituberculosa convencional en adultos VIH-positivos con TBC pulmonar no tuvo efectos significativos sobre la mortalidad, la conversión del cultivo a los 2 meses ni los resultados bacteriológicos a los 12 meses. (Mwinga A, Nunn A, Ngwira B, Chintu C, Warndorff D, Fine P, et al. Mycobacterium vaccae (SRL172) immunotherapy as an adjunct to standard antituberculosis treatment in HIV-infected adults with pulmonary tuberculosis: a randomised placebo-controlled trial. Lancet 2002;360:1050-1055.)

\section{Sistemas de sujeción para niños en los automóviles}

En Canadá, los accidentes de tráfico constituyen la principal causa de muerte en la población infantil. El hecho de no usar sistemas de sujeción o de no usar el sistema adecuado se asocia frecuentemente a las muertes y lesiones por esta causa. Teniendo en cuenta estos datos, la información proporcionada por el médico a los padres acerca de la importancia del uso de sistemas de sujeción adecuados puede ser la actividad más importante para la promoción de la salud infantil. En este artículo el autor resume los conocimientos actuales sobre el uso de sistemas de sujeción para niños en los automóviles.

Las autoridades rodoviarias canadienses recomiendan cuatro tipos de sistemas de sujeción para niños, dependiendo del peso: 1) asientos de seguridad que miren hacia atrás para los niños de hasta $10 \mathrm{~kg}$ (aproximadamente 1 año); 2) asientos de seguridad que miren hacia delante para los niños de 10 a 18 o $22 \mathrm{~kg}$ (aproximadamente 4,5 años; 3) asientos elevados para niños de 22 a $27 \mathrm{~kg}$ (aproximadamente 8 años), y 4) uso del cinturón de seguridad en el asiento trasero del automóvil para niños de más peso. Estas cifras de peso son variables según los países.

El asiento trasero es el más seguro. Según un estudio, el ir sentado en el asiento trasero reduce en $36 \%$ el riesgo de muerte de niños implicados en accidentes mortales, independientemente del uso, o no, de sistemas de sujeción. Según otro estudio, el riesgo de sufrir lesiones graves o mortales es 1,7 veces menor (intervalo de confianza de 95\%: 1,6 a $2,0)$ cuando el niño va sentado en el asiento trasero que cuando va en el delantero. Otros estudios han estimado que el uso correcto de asientos de seguridad para niños reduce el riesgo de muerte en $71 \%$ y el riesgo de lesiones graves en $67 \%$, y que el uso de asientos de seguridad que miran hacia atrás reduce en $96 \%$ las lesiones en menores de 3 años.

La frecuencia del uso de sistemas de sujeción disminuye a medida que aumenta la edad de los niños. En Canadá, en 1997, dicha frecuencia fue de 96\% para los lactantes, de $91 \%$ para los niños de 1 a 4 años, y de 85\% para los de 5 a 15 años. En los Estados Unidos de América (EE. UU.), las cifras correspondientes fueron $85 \%, 60 \%$ y $65 \%$ en 1996 . El factor que mejor predice el uso de sistemas de sujeción para niños en accidentes mortales es el uso de dichos sistemas por el conductor del vehículo. Por otra parte, la frecuencia del uso inadecuado de estos sistemas ha oscilado entre 44 y $81 \%$ para los asientos de seguridad para niños, y entre 33 y $50 \%$ para los asientos elevados.

La American Academy of Pediatrics ha recomendado recientemente que los niños deben ir en asientos que miren hacia atrás durante el máximo tiempo posible, y al menos hasta que cumplan 1 año. Muchos de estos asientos están diseñados para niños de hasta $14 \mathrm{~kg}$. Esta posición es segura siempre que haya apoyo para la cabeza, y todavía no se han descrito casos de lesiones neurológicas catastróficas en niños sentados así.

La tríada completa de las lesiones producidas por los cinturones de seguridad consiste en equimosis de la pared abdominal, lesiones de las vísceras abdominales y fracturas vertebrales. Los niños son más propensos a estas lesiones porque a menudo el cinturón les queda grande y les pasa por el abdomen, en vez de por las caderas. Este problema se resuelve con los asientos elevados, cuyo uso es, no obstante, muy escaso en todos los países.

Los niños sujetados por cinturones mal ajustados o sentados en sillas de seguridad para niños mal sujetadas corren el riesgo de salir despedidos del vehículo, sobre todo en caso de vuelco. Aunque no hay datos específicos referidos a los niños, en adultos, el salir despedido del vehículo multiplica por ocho el riesgo de muerte. La mala sujeción de los niños es un problema endémico, y es así que los vuelcos son la causa de $29 \%$ de los accidentes mortales para niños en los EE. UU., aunque solo representan 1 a $2 \%$ de los accidentes.

Las colisiones laterales son peligrosas para los niños. En un estudio, se produjeron lesiones importantes en $41 \%$ de las colisiones laterales, en $15 \%$ de las colisiones frontales y en $3 \%$ de las colisiones por detrás en las que se vieron implicados niños de edad escolar que estaban usando sistemas de sujeción. En otro estudio, la mortalidad de los niños en las colisiones laterales fue de $30 \%$, frente a $17 \%$ en las frontales. Como los sistemas de sujeción no alteran de forma significativa la probabilidad de sufrir lesiones en una colisión lateral ni su gravedad, se recomienda 
situar al niño en el asiento central trasero, siempre que sea posible, y ajustar bien los cinturones para reducir la movilidad lateral de la cabeza.

Los niños sentados en el asiento delantero pueden morir o sufrir lesiones graves provocadas por las bolsas de aire (air bags), incluso en accidentes menores. El inflado instantáneo de las bolsas se ha equiparado a una colisión a $300 \mathrm{~km} / \mathrm{h}$. Los niños situados en la zona de inflado de la bolsa pueden sufrir lesiones craneales y medulares mortales. Los niños de menos de 13 años deben ir en el asiento trasero, lejos de las bolsas de aire. La experiencia actual con las bolsas de aire laterales es todavía escasa. Las bolsas de aire traseras pueden causar lesiones graves a niños de hasta 6 años. Es recomendable evitar las bolsas de aire traseras en automóviles en los que viajen niños $\mathrm{y}$, de hecho, en los EE. UU., la política actual es vender los automóviles con estas bolsas desactivadas.

Los modelos de automóviles más recientes disponen de nuevos sistemas que facilitan la instalación de los asientos de seguridad para niños (sistemas LATCH o isofix) y proporcionan un anclaje más seguro que los cinturones de seguridad.

En resumen, los niños deben sentarse en el asiento trasero del automóvil, deben ir sujetos por un dispositivo apropiado para su edad y peso que esté fijado adecuadamente, y deben sentarse lejos de las bolsas de aire. (Howard AW. Automobile restraints for children: a review for clinicians. CMAJ 2002;167:769-773.)

\section{La obesidad en los adultos de los Estados Unidos (1999-2000)}

En Estados Unidos de América (EE. UU.) se vienen realizando encuestas nacionales de salud y nutrición (National Health and Nutrition Examination Surveys) desde la década de los sesenta. La tercera de estas encuestas (NHANES III; 1988-1994) reveló que la prevalencia de la obesidad, definida como un índice de masa corporal (IMC) $\geq 30$, había aumentado en cerca de $8 \%$, después de haberse mantenido relativamente estable entre 1960 y 1980. En este artículo se describen los resultados de la última NHANES (1999-2000) con respecto a las tendencias de la prevalencia de la obesidad y de la distribución de frecuencias del IMC.

Los procedimientos utilizados en la selección de la muestra y en la realización de las entrevistas y los exámenes fueron similares a los de las encuestas anteriores. Este informe se basa en los datos de 4115 adultos de ambos sexos. El sobrepeso se definió como un IMC $\geq 25$, la obesidad como un IMC $\geq 30$ y la obesidad extrema como un IMC $\geq 40$.
La prevalencia de la obesidad se mantuvo relativamente constante entre 1960 y 1980, pero aumentó en la NHANES III (1988-1994). Los datos más recientes (NHANES 1999-2000), muestran un nuevo aumento de la prevalencia de la obesidad en ambos sexos y en todos los grupos de edad.

La prevalencia de la obesidad ajustada por edad fue de 30,5\% en la NHANES 1999-2000, frente a $22,9 \%$ en la NHANES III (1988-1994) $(P<0,001)$. La prevalencia del sobrepeso también aumentó del 55,9 a $64,5 \%(P<0,001)$, al igual que la de la obesidad extrema, de 2,9 a $4,7 \%(P=0,002)$.

Los aumentos observados entre la NHANES II (1974-1980) y la NHANES III (1988-1994) fueron estadísticamente significativos en todos los grupos de edad en ambos sexos, y lo mismo ocurrió con los aumentos observados entre la NHANES III (1988-1994) y la NHANES 1999-2000; la única excepción fue el grupo de hombres de 40 a 59 años, en el que también se observó un aumento, pero no significativo. El aumento observado entre la NHANES III (1988-1994) y la NHANES 1999-2000 fue casi tan grande como el observado entre la NHANES II (1974-1980) y la NHANES III (1988-1994).

La prevalencia de la obesidad y de la obesidad extrema también aumentó entre la NHANES III (1988-1994) y la NHANES 1999-2000 en todos los grupos étnicos analizados: blancos no hispanos, negros no hispanos y estadounidenses de origen mexicano. En general, los aumentos fueron similares en los tres grupos, aunque hubo una tendencia no significativa hacia un mayor aumento en las mujeres negras no hispanas. Más de 50\% de las mujeres negras no hispanas de 40 años o más eran obesas, y más de $80 \%$ tenían sobrepeso.

También se analizó la distribución poblacional del IMC. En los hombres de 60 a 79 años, dicha distribución se desplazó a la izquierda entre la NHANES III (1988-1994) y la NHANES 1999-2000, pero el desplazamiento fue mayor en los percentiles más altos de la distribución. También se observaron cambios similares en los hombres y mujeres de 20 a 39 y 40 a 59 años. En las mujeres de 60 a 79 años los desplazamientos fueron más uniformes.

El aumento de la prevalencia del sobrepeso y la obesidad plantea interrogantes importantes sobre las implicaciones sanitarias de esta tendencia, dado que la obesidad es un factor de riesgo de muchas enfermedades crónicas, como la diabetes, la hipertensión, la hipercolesterolemia, los accidentes vasculares cerebrales, las cardiopatías, algunos cánceres y las enfermedades articulares. De todas estas enfermedades, la diabetes podría ser la más estrechamente relacionada con la obesidad y, de hecho, su prevalencia parece haber aumentado a medida que ha aumentado la prevalencia de la obesidad. En 